\title{
Towards Process Tomography for Monitoring Pressure Filtration
}

\author{
Trevor A. York, Member, IEEE, John L. Davidson, Lukasz Mazurkiewich, Reginald Mann, and Bruce D. Grieve
}

\begin{abstract}
This paper reports on progress towards the first continuous application of electrical impedance tomography to a production scale industrial process. It includes the design and implementation of the worlds first certified intrinsically safe electrical tomography system. Zener barrier (ZB) modules and intrinsically safe relays provide electrical isolation and the instrument is certified for operation in a Zone 0 environment. Two systems have been operating successfully on production pressure filters for two years. A sensor architecture has been implemented that is compliant with the process such that it is not detrimental to efficiency or the integrity of the associated vessel structure. The MATLAB-based EIDORS three-dimensional software toolkit has been employed to yield images from simulated data. To overcome problems that arise due to dramatic changes in the conductivity of materials in the vessel during the process, a new approach is introduced that proposes the use of dynamic sensitivity maps that evolve during the batch based on the predicted mean conductivity at each stage. Real data present challenges due to a number of factors, notably the $\mathrm{ZB}$ modules that are integral to the intrinsically safe instrumentation. However, a simple approach to data processing has yielded process relevant information using real tomographic data.
\end{abstract}

Index Terms-Electrical tomography, image reconstruction, intrinsic safety, pressure filtration.

\section{INTRODUCTION}

$\mathbf{P}$ RESSURE filtration is a generic process that is carried out across the chemical industry for the separation of a liquid from a solid phase. The desired product may be present in either the liquor or suspension and is often generated within the same vessel, for example, as the result of a reaction stage or purification via crystallization. For solid products, it is common for the removal of mother liquor to be followed by a number of solvent washes and/or drying with an inert gas stream. This may be a lengthy procedure extending to a number of hours or days. Pressure filtration vessels can range in size from liters to

Manuscript received December 17, 2003; revised October 14, 2004. This work was supported in part by the U.K. Department of Trade and Industry under Grant YAF 8/2/3228 and in part by the U.K. Engineering and Physical Science Research Council under Grant GR/R21745 under the LINK "Sensors and Sensor Systems for Industrial Applications" program. The associate editor coordinating the review of this paper and approving it for publication was Dr. Krikor Ozanyan.

T. A. York and J. L. Davidson are with the School of Electrical and Electronic Engineering, University of Manchester, Manchester M60 1QD, U.K. (e-mail: t.york@manchester.ac.uk; j.davidson-2@manchester.ac.uk).

L. Mazurkiewich is with the Computer Engineering Department, Technical University of Lodz, 90-924 Lodz, Poland (e-mail: 1mazurk@writeme.pl).

R. Mann is with the School of Chemical Engineering and Analytical Science, University of Manchester, Manchester M60 1QD, U.K. (e-mail: r.mann@manchester.ac.uk).

B. D. Grieve is with Global Specialist Technology Department, Syngenta, Huddersfield, HD2 1FF, U.K. (e-mail: bruce.grieve@ syngenta.com).

Digital Object Identifier 10.1109/JSEN.2004.842631 tens of cubic meters and on-line monitoring is usually limited to measurements of temperature, pressure, and level. Research into the application of electrical impedance tomography (EIT) to pressure filtration was initiated in 1996 under a U.K. Government-funded Technology Foresight Challenge project which aimed to evolve industrial tomography from the research laboratory to the production environment. The Foresight project was based on two $1.5-\mathrm{m}^{3}$ units within Syngenta (formerly Zeneca) and has been previously reported [1], [2]. This early work has led to the PROCEMON project, which is the subject of this paper.

During the course of the PROCEMON project, a number of industrial challenges have been addressed in order for the instrumentation to be deployed on two full-scale production vessels at Syngenta's Manufacturing Centre in Huddersfield, U.K. This has resulted in the first intrinsically safe electrical tomography system that is certified for use within a Zone 0 hazardous atmosphere [3]. It has also been necessary to utilize electrode materials and array structures which are compatible with the unit operation [4], [5]. Access to the filtration units is only possible during annual maintenance, and this has imposed challenges for commissioning the electrodes. In order to avoid compromising vessel integrity the resulting electrode arrays are unusual in that they are located on the base of the filter rather than the conventional arrangement, in a circular ring around the walls. Consequently, the larger of the two demonstrators, and the subject of this paper, employs 24 electrodes arranged as two concentric rings within the plane of the filter cloth. The $36-\mathrm{m}^{3}$ filtration unit, having diameter $4 \mathrm{~m}$ and height about $2.8 \mathrm{~m}$, and electrode arrangement are shown in Figs. 1 and 2. A further challenge has been to provide on-line results across the company intranet such that they are readily accessible to plant operators.

A key aim of PROCEMON is to monitor on line the homogeneity and solvent distribution within the filter cake during the separation, washing, and drying stages, using three-dimensional (3-D) image reconstruction. The geometry of the electrode arrangement together with the intrinsically safe instrumentation present some new challenges compared to traditional applications. Image reconstruction algorithms employ a sensitivity map to represent the influence that the material in each pixel has on each measurement. The simplest algorithm, linear back projection, produces qualitative images by linearizing the problem. Quantitative images can be obtained using iterative algorithms [6]. EIDORS 3-D [7] is a toolkit of MATLAB functions and offers the ability to explore electric field distributions using finite-element modeling, to generate sensitivity maps that depend on the application geometry and to implement a variety of reconstruction algorithms. The toolkit has been employed in the 


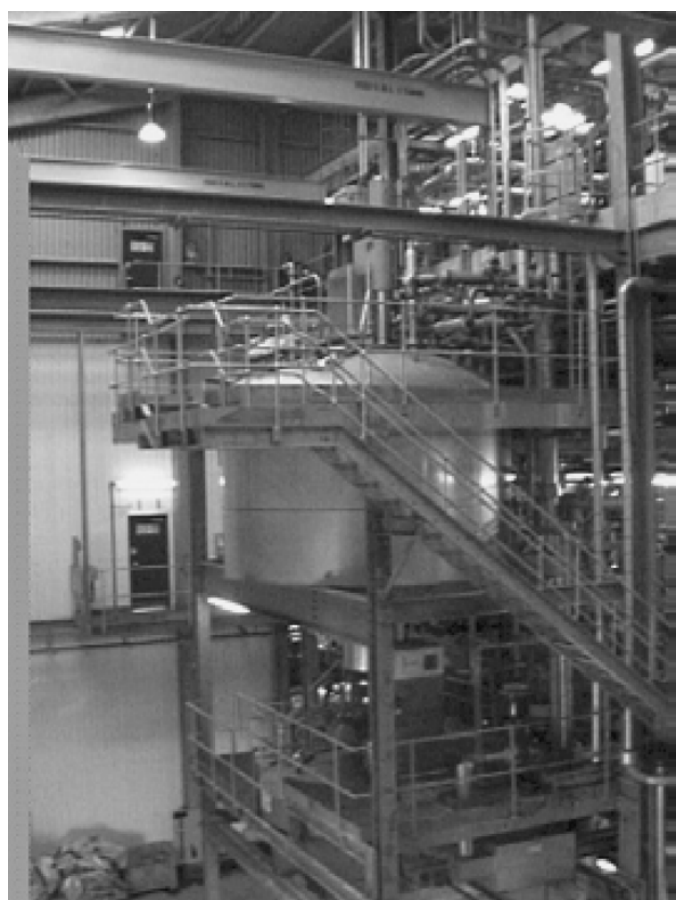

Fig. 1. Photograph of a $36-\mathrm{m}^{3}$ vessel.

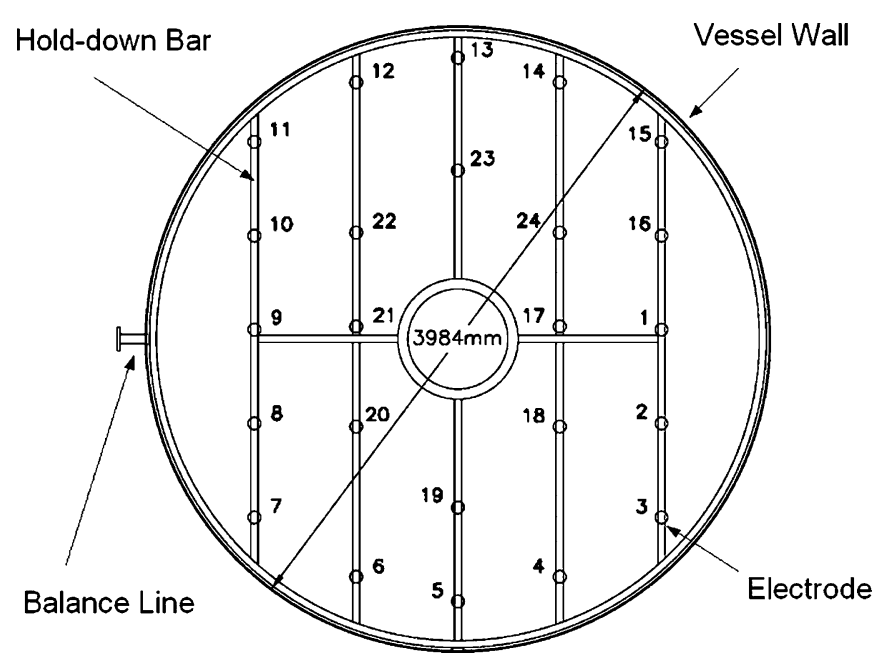

Fig. 2. Diagram showing arrangement of electrodes.

present work to explore 3-D image reconstruction. The issues introduced above are discussed in more detail in the following sections.

\section{INTRINSICALLY SAFE TOMOGRAPHY SYSTEM}

\section{A. Electrical Tomography}

Electrical tomography systems [8] typically comprise sensors, measurement and switching electronics, signal conditioning, analog/digital conversion, communications, and a computer hosting control and data processing, including inversion, analysis, and display algorithms. A schematic representation of a typical system is shown in Fig. 3.

For capacitance and inductance systems, the electrodes are frequently noninvasive. For resistance measurements, the electrodes are usually invasive but not intrusive. However, for ves-

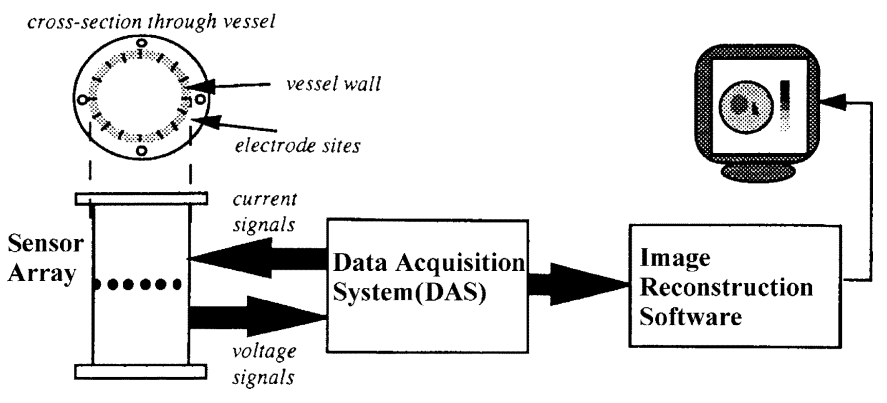

Fig. 3. Schematic representation of electrical tomography system.

sels with conducting walls, it is necessary in all cases for the electrodes to be located inside the vessel. Signals from the sensors are routed to the measuring electronics by a multiplexer which is usually implemented using solid state switches. Parasitics that are associated with these switches are particularly important, affecting switching speed and noise and selection of appropriate devices is an important decision. When the initial signals have been amplified and buffered programmable gain and offset are usually employed, to accommodate a wide range of signals, with demultiplexing and filtering in analog hardware. The analog signals are converted to digital format and transferred to the host, usually via a high-speed, robust, serial communications link. Typically, data rates of about $10 \mathrm{Mbits} / \mathrm{s}$ have been desirable. It is also becoming increasingly common for systems to have embedded microcontrollers, which relieve the host processor of some of the supervisory tasks in order to focus on data processing.

The 36- $\mathrm{m}^{3}$ filter that is the subject of PROCEMON has a batch time of about $50 \mathrm{~h}$ and an image rate of $1 / \mathrm{min}$ is entirely acceptable. Therefore, an electrical tomography system was developed to explore the potential advantages of using a commercial inductance-capacitance-resistance (LCR) meter, namely that it can provide robust impedance measurements across a range of frequencies. The HP 4284A LCR meter can provide four terminal electrical impedance measurements (I+, $\mathrm{I}-, \mathrm{V}+, \mathrm{V}-$ ) in the frequency range $20 \mathrm{~Hz}$ to $1 \mathrm{MHz}$. A cross-bar switch allows the four terminals on the LCR meter to be connected to any electrode under the control of a PC. Configuration of the cross-bar switch is achieved by downloading serial data bits to a shift register. These data bits control relays which are able to switch fast enough to satisfy the demands of many batch processing applications including pressure filtration. A schematic diagram, representing a four-electrode system is shown in Fig. 4. This comprises 16 switching modules (R1 to R16), each controlled by a bit of the shift register which is daisy-chained between the modules. Prior to the development of the intrinsically safe system, each switch was implemented using a mercury-wetted relay (Clare MSS21A05B). Relays were selected in order to reduce parasitic switching impedance. A frame of data from a 16-electrode tomography system can be acquired in about $30 \mathrm{~s}$ using this system. The intrinsically safe tomography system described in the next section is based on this design and it satisfies the crucial requirement that it must be able to operate in a hazardous area.

For applications in which the process vessels have conducting walls, it is preferable to adopt a "metal-wall" measurement 


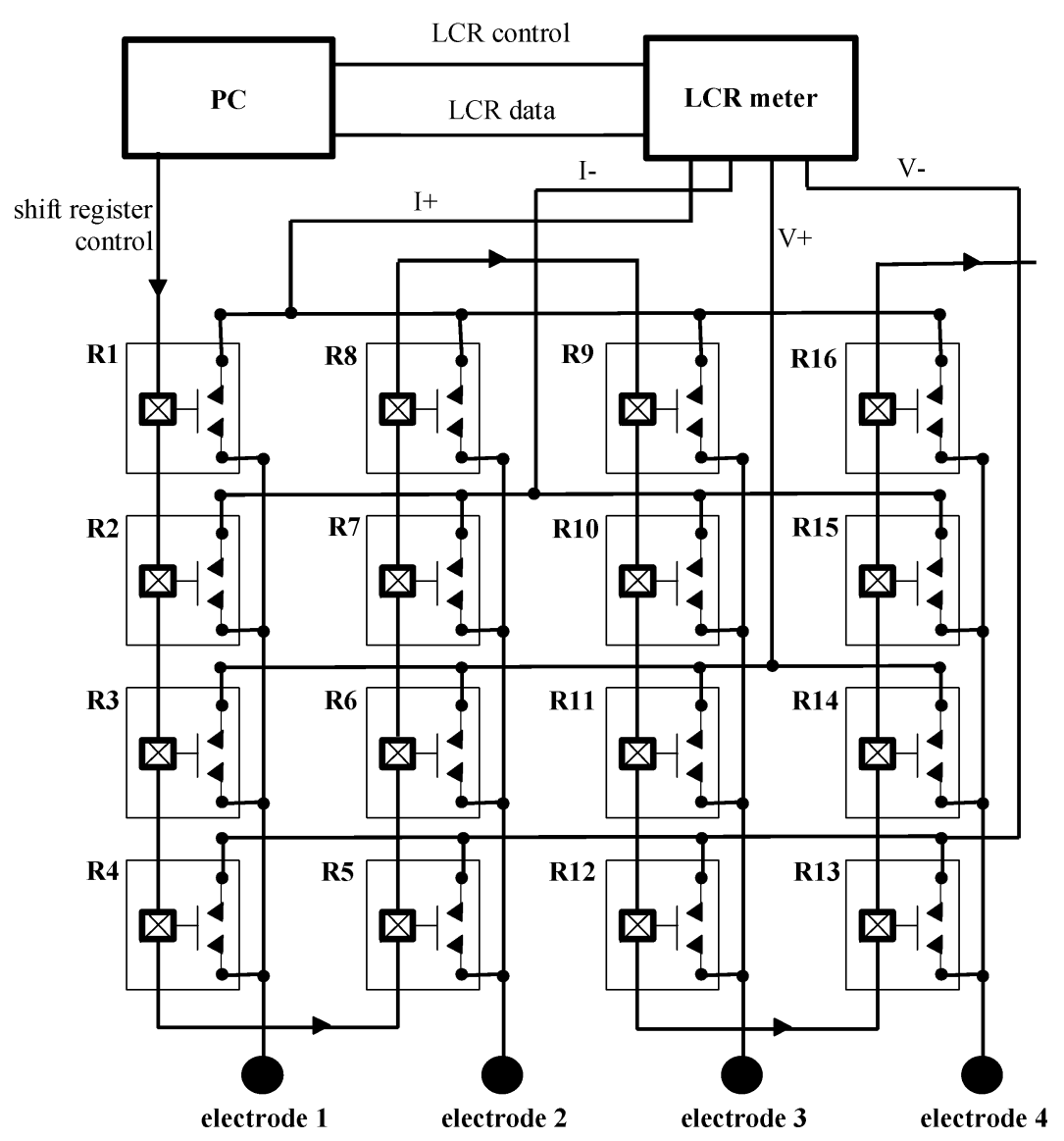

Fig. 4. LCR-based tomography system.

strategy in order to improve measurement sensitivity [9]. The benefits of this strategy have been established using finite-element modeling. In the metal-wall strategy, current is sourced on one electrode and sunk to earth. The potential is measured on all other electrodes and referenced to earth. This strategy is employed in the intrinsically safe instrument that is described here. To implement this strategy the LCR meter is configured in a three-terminal input mode, the effects of which will be discussed in Section V.

\section{B. Intrinsically Safe System}

Across the process sector, many organic solvents and products are commonly used which are flammable in air or other gas mixtures. To allow electrical apparatus to be applied within such an environment, a branch of engineering has been developed to classify the risk and reduce the probability of an ignition source being present [10]. This methodology is a legal requirement in Europe and industrial nations elsewhere.

There are a number of ways of ensuring that a flammable atmosphere is isolated from any significant energy source, however, by its very nature electrical tomography requires energy to be injected into a potentially flammable atmosphere and therefore only one approach, intrinsic safety (I.S.), is appropriate. Conveniently, of all the protection methods, I.S. certification has the greatest degree of integrity and is, therefore, the most appropriate for Zone 0 , which is the most stringent classification of hazardous environment suggesting an area in which an explosive gas/air mixture is continually present or is present for long periods.

I.S. certification relies on constructing apparatus in such a manner that the maximum electrical energy that is injected is less than the minimum ignition energy of the hazardous environment. This requirement needs to be satisfied for both normal operation and for worst case failure. In realizing an I.S. version of the tomography system it is necessary to acknowledge the demands of certification, not only in terms of cost, but also the time delay between concept and approval. For both of these reasons, for the present system, it was decided to simplify the problem by locating only passive electrical components in the hazardous, Zone 0, environment found within the pressure filter. In other words, only the electrodes and connecting wires are located in the hazardous area. All active, electronic components and power supplies are located on the safe side of the barrier. By limiting the dimensions of the electrodes and the maximum capacitance and inductance of the interconnecting wires, it has been possible to define the equipment within the hazardous area as "simple apparatus" and allow up to $50 \mathrm{~m}$ of co-axial cable to be connected to each of the electrodes. For an intrinsically safe system, it is necessary to define a boundary between the hazardous and nonhazardous areas. In the present system, all of the interface electronics and the control computer are mounted remotely in the plant switch room, which is a safe area some $50 \mathrm{~m}$ from the filter. 


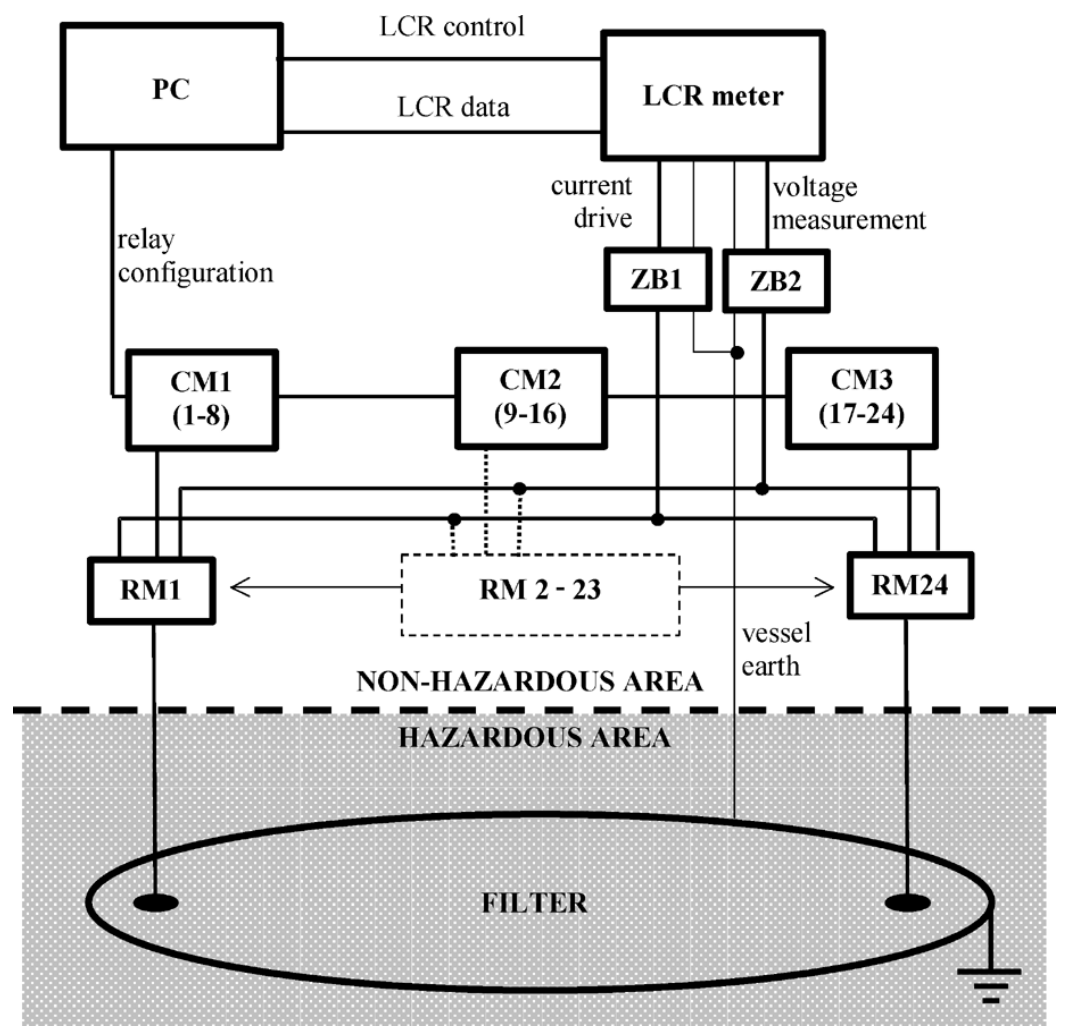

Fig. 5. Schematic of a 24-electrode intrinsically safe tomography system.

Building on the original tomography system described above, the LCR instrument has been combined with a custom switch matrix and image reconstruction software to produce a new tomographic system which conforms to the requirements of I.S. certification. The philosophy behind the design of the I.S. system has been to utilize, wherever possible, existing certified components. This is achieved by taking an existing system certification for a typical Zener barrier (ZB) in a strain gauge configuration and expanding on this using a series of certified I.S. relay modules (RM), as shown in Fig. 5. ZB1 (Pepperl+Fuchs, p/n Z966) and ZB2 (Pepperl+Fuchs, p/n Z964) are intrinsically safe ZB modules (ZB), certified for Zone 0 . Each relay module (RM) is configured via a control module (CM) and connects to an electrode to determine whether it provides current to the filter; a measurement to the LCR or is disconnected. Relay modules RM2 to RM23 are omitted for clarity. The function of the ZB modules is to limit the power that may be delivered to the hazardous area to an acceptable level even if all the electrodes are directly shorted together.

For all measurements, the LCR meter delivers a drive signal, in the form of a current that is returned to earth, to a selected electrode via ZB1, and measures a voltage, relative to earth, on another selected electrode via ZB2. The driven and measurement electrodes are selected by a matrix of relay modules in a manner that prevents inadvertent connection to additional energy sources. Perhaps a more obvious solution may be to employ a ZB for each electrode such that the switching circuitry does not need to be certified, as it can be located on the safe side of the system. However, such an arrangement presents some limitations for the current drive capacity for each electrode. Consider a situation where all electrodes are driven at the same time. The currents that are supplied by the ZBs will be added together, and, consequently, for such an arrangement, the drive capacity of each ZB must be reduced in order to ensure that the overall current is kept within limits. In addition, unlike the present system, the maximum drive current for each ZB will depend on the number of electrodes. For instance, in a 16-electrode system, a drive capacity of $1 \mathrm{~mA}$ from each ZB could deliver a total of $16 \mathrm{~mA}$ to the process if all electrodes were driven at the same time. Similarly, 32 electrodes could deliver $32 \mathrm{~mA}$, and so on. In the system described here, the maximum current that can be delivered is not dependent on the number of electrodes.

Although the relays that have been used are commercially available, intrinsically safe components (Pepperl+Fuchs, p/n KFD0-RO-Ex2), it has been necessary to design a bespoke control module (CM1, CM2, and CM3 in Fig. 5) to provide them with I.S. control signals to ensure that the power to these components is constrained to that defined within the relevant certificate. Each control module provides intrinsically safe control signals to determine the state of eight relay modules and, therefore, three control modules are required to configure 24 electrodes. These signals are derived from a shift register in each control module. The shift registers are "daisy-chained" between the control modules and are driven directly from the PC interface. Zener diodes in the CM limit the voltage excursions on the control signals to satisfy the demands of intrinsic safety. As the system is designed to operate in accordance with the EEx-ia code (suitable for European Zone 0), all the circuitry connected to the potentially flammable atmosphere must be capable of having up to two faults, while still maintaining the integrity of the I.S. signal.

The whole system is controlled by software that communicates with the LCR meter via IEEE 488 and with the switching matrix via a digital port. To attain approval for an I.S. design, 


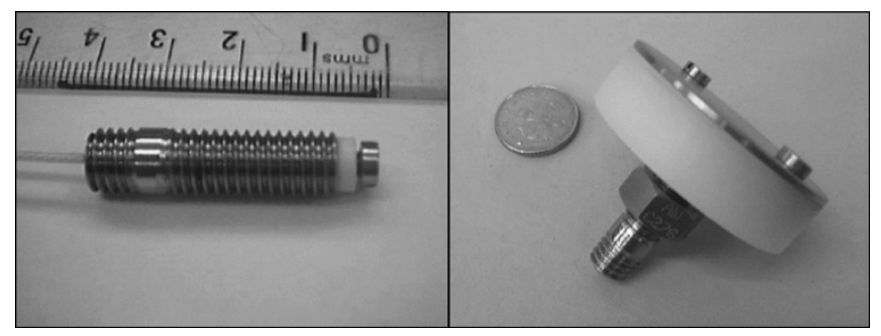

Fig. 6. Mark II electrode detail for $36-\mathrm{m}^{3}$ filter vessel.

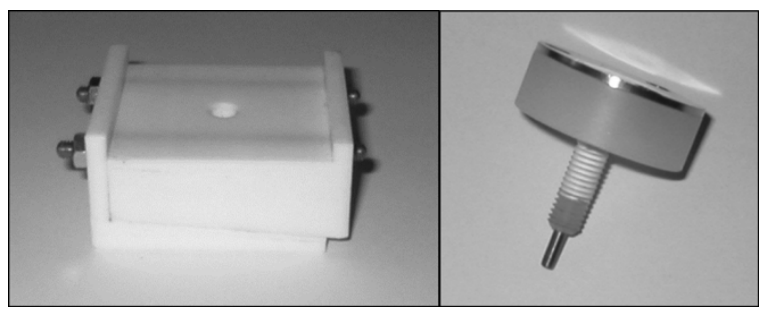

Fig. 7. Mark III electrode detail for $36-\mathrm{m}^{3}$ filter vessel.

it is necessary to have it endorsed by a registered independent authority. This process requires both the circuit theory and the practical implementation to be scrutinized, including such parameters as printed circuit board (PCB) track thickness, routing of I.S. and non-I.S. cabling, security of construction, creepage and clearance distances, and electrode dimensions. As a result of these considerations, for the present system, the electrodes must be no more than $72 \mathrm{~mm}$ in diameter with a minimum separation of $8 \mathrm{~mm}$ from the ground plane in the filter. For the purposes of the project, SIRA Test and Certification, Ltd., Chester, U.K., were engaged as the approval authority.

\section{Electrode Design AND COMMISSIONING}

PROCEMON has concentrated on two prototype systems located within manufacturing plants at Syngenta. The $36-\mathrm{m}^{3}$ filter application was originally commissioned in October 2000 and has undergone four iterations of electrode design, which have been installed during each annual maintenance period. The aim has been to evolve a sensor which is simple to fit and replace by maintenance personnel, does not require any additional drillings into the fabric of the vessel, is tolerant to the harsh conditions within the process, and introduces imperceptible changes to the overall operation of the unit. The original Mark I electrode design has been previously reported along with its failure mechanisms [4]. Figs. 6-8 give details of the subsequent Mark II to IV electrodes, the last of which was commissioned in the September 2003 plant shut down. Though the structure of these have been altered from one version to the next their arrangement within the vessel, as two concentric distorted rings of 8 and 16 electrodes within the plane of the filter cloth and on top of the cloth hold-down bars, has been fixed.

In summary, the Mark II electrode overcame the connector weaknesses of the Mark I by using the head of a hastelloy screw, to which the cabling was attached at the lower end via a PTFE insert and resin seal, to make contact with a removable hastelloy electrode plate. This unit provided consistent measurements but suffered from the mechanical failure of the

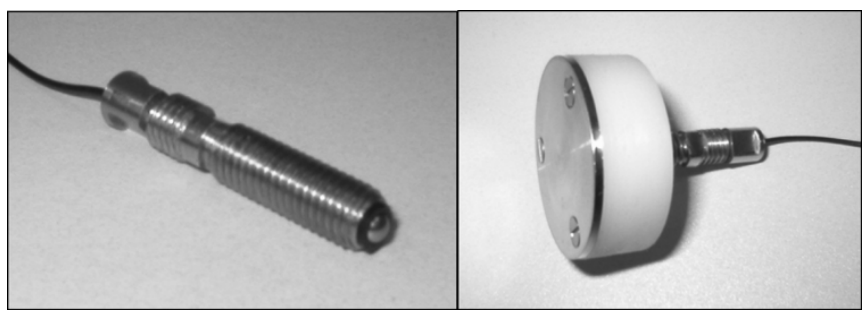

Fig. 8. Mark IV electrode detail for $36-\mathrm{m}^{3}$ filter vessel.
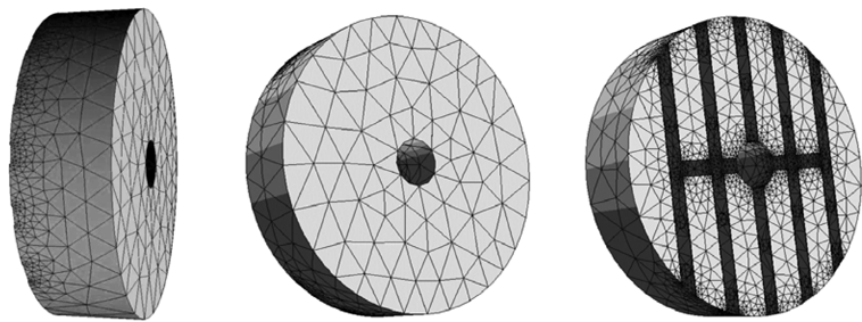

Fig. 9. Three views of the 3-D mesh including electrodes, hold-down bars, and central pillar.

insulating insert, eventual seepage of process liquid in from the cable end, and a tendency for the stud assembly to be damaged during filter maintenance due to its intrusion above the height of the filter cloth level. The Mark III electrode again provided reliable readings, this time through locating a connector plate under the cloth hold-down bar so that it would be protected from damage and could incorporate a proprietary compression sealing system around the cable entry point. The removable electrode, comprising an electrode plate in conjunction with a sprung plunger, was then screwed into the hold-down bar to make contact with the connector plate. The principal weakness here was associated with flooding of the connection point with process liquor on removal. The Mark IV design is a distillation of all the acquired practical experience and reverts to an intrusive hastelloy stud assembly which is stronger than the earlier versions by virtue of utilizing a commercial sprung ball connector. This miniature component has the double benefit of requiring only a narrow bore drilling to be made through the stud while also taking up any tolerance in the electrode plate positioning. The cable entry point is secured through a custom compression fitting based on a proven design.

\section{IMAGE RECONSTRUCTION USING EIDORS 3-D}

\section{A. Filter Modeling}

The finite-element method (FEM) is a popular numerical technique for solving electric field equations [11], and this has been used extensively in industrial process tomography. Although there are many commercial software applications for FEM modeling, high flexibility is required for tomography applications, and this is not available in commercial software. For the present project, the EIDORS 3-D toolkit has been used [7]. This publicly available software runs in a MATLAB environment and comprises a library of functions which enable image reconstruction algorithms to be explored. Mesh generation for finite-element modeling has been realized using NetGen [12]. Equations must be defined for each element and 
a)

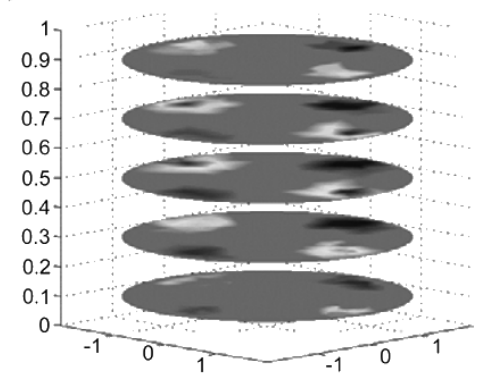

Reconstructed image

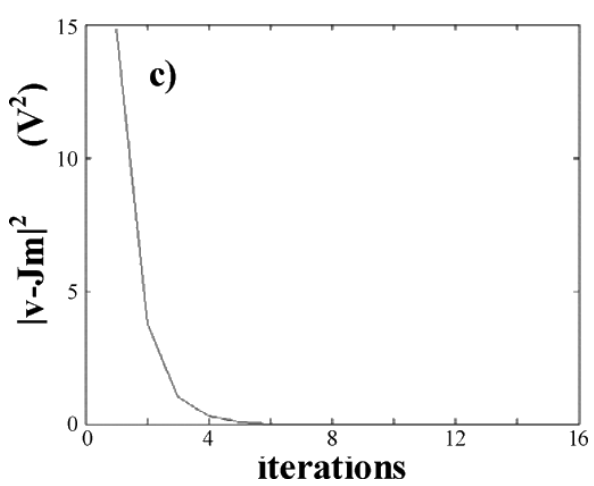

b)
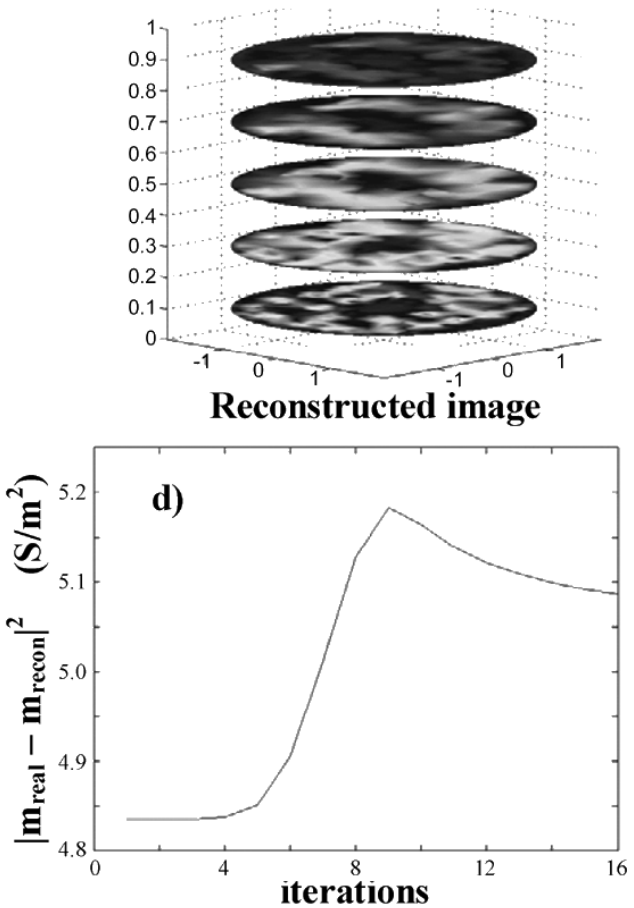

Fig. 10. Results of reconstruction using a fixed sensitivity matrix.

boundary conditions specified. As resistance tomography and the metal-wall strategy have been used [9] the injected current, fixed potential boundary conditions and electrode impedance model have been applied. The equations can be expressed in form

$$
Y V=I
$$

where $Y$ is the stiffness matrix that depends on conductivity distribution, element shapes, and boundary conditions, $V$ is the potential vector, which represents electric field distribution, and $I$ is a forced matrix which represents the known currents and potentials.

In order to predict accurate values, it is important to embrace all geometric details in the model. For electrical impedance tomography, all conducting surfaces should be included as they have a significant influence on the electric field distribution. Pressure filters usually house a selection of metal "furniture" and in the present case this includes an agitator, clamping bars to hold the filter cloth and a central pillar. The 24 electrodes have been modeled as cylindrical conductors located on the base of the filter. The resulting 3-D mesh is shown in Fig. 9 and consists of about 6000 tetrahedral elements. The electrodes are just discernible as patches of dense mesh on the clamping bars which are visible as straight "fingers." The hole in the center is due to the presence of a large metal pillar in the filter vessel. Using an Athlon 1.7XP, the model is solved in about $8 \mathrm{~s}$ to yield the distribution of electric field and boundary voltages.

\section{B. Image Reconstruction}

Almost all algorithms for reconstructing images of conductivity use a linearization technique [6], which leads to the fol-

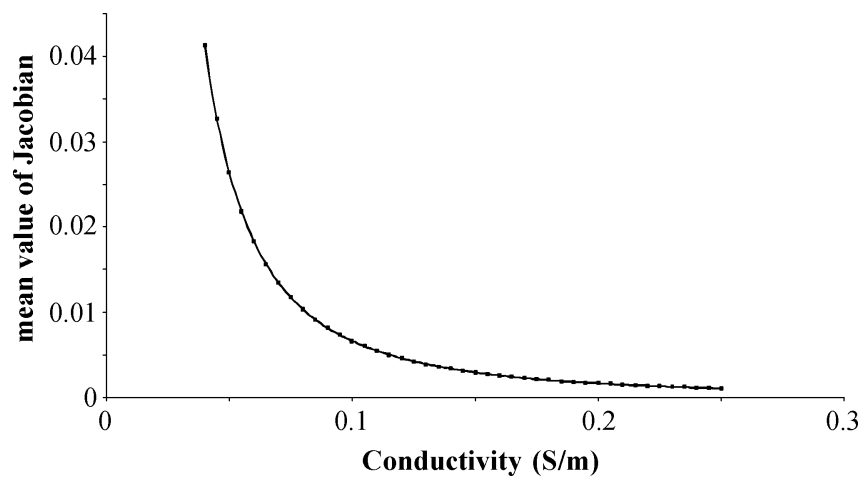

Fig. 11. Mean value of sensitivity matrix as a function of material conductivity.

lowing equation to calculate the expected change in measurement value:

$$
\partial v=J \partial m
$$

where " $v$ " is the measurement, " $m$ " is material conductivity, and " $J$ " is the sensitivity matrix. During reconstruction, the aim is to find the " $m$," which minimizes the following:

$$
\|v-J m\|^{2}
$$

It is clear that, in an ideal case, a different norm should be minimized, e.g.,

$$
\left\|m_{\text {real }}-m_{\text {recon }}\right\|^{2}
$$

or

$$
\max \left(\left|m_{\text {real }}-m_{\text {recon }}\right|\right) \text {. }
$$




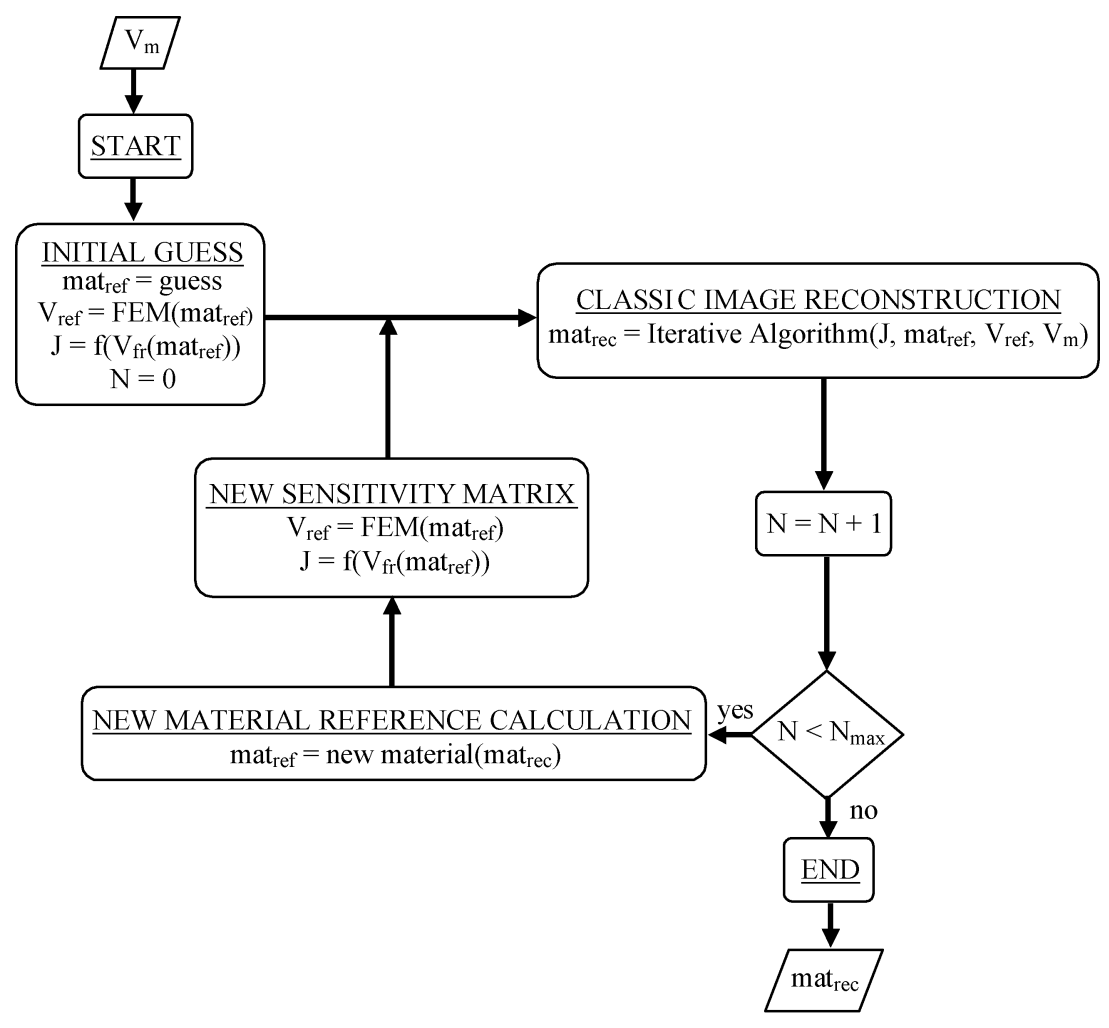

Fig. 12. Quantitative reconstruction algorithm with the evolving sensitivity matrix.

In practice, this is not possible, since " $m_{\text {real }}$ " is unknown. In fact, the electric field is nonlinear and the sensitivity matrix " $\mathrm{J}$ " is correct only for one unique distribution of conductivity. In many applications, the conductivity distribution remains close enough to the reference for the sensitivity matrix to be appropriate. However, during filtration processes, such as those considered here, the conductivity may change over a wide range and the sensitivity matrix may be far from ideal, leading to poor-quality images. In this work, the iterative Landweber algorithm has been used for image reconstruction [6]. Fig. 10 demonstrates this effect for simulated data in the filter model described above. Fig. 10(a) shows the resulting 3-D images for the case where the conductivity is close to the reference. Four phantoms are clearly visible, two with elevated conductivity appearing as light patches and two with lowered conductivity appearing as dark patches. However, in Fig. 10(b), where the conductivity is not close to the reference, the image is seriously degraded and the phantoms are not visible. Fig. 10(c) and (d) shows the evolution of the functions in (3) and (4), respectively, during the reconstruction shown in Fig. 10(b). Clearly, the value of $\left|m_{\text {real }}-m_{\text {recon }}\right|^{2}$ does not converge, and this is typical of the results that we have observed during this work. This arises due to the strong dependence of the sensitivity matrix as a function of material conductivity, as shown in Fig. 11.

From the discussion presented above, it is suggested that the " $J$ " matrix should evolve during processing and be repeatedly calculated for a material distribution that is close to the actual distribution at each step. An algorithm to achieve this is shown in Fig. 12.

An initial guess is made regarding the material distribution, and this is used to generate a reference sensitivity matrix. Iter- ative image reconstruction is then performed on a set of measurement data using the reference matrix. At the end of this iterative step, the resulting distribution of material that is predicted from the reconstruction is used to derive a new matrix for the next iterative step on new measurement data. This process is repeated for each subsequent data set. In practice the problem is strongly nonlinear and reconstruction can introduce significant error after only a small number of measurement sets. However, our work suggests that although the reconstructed distribution may be considerably different from the actual distribution the general trend reflects the real situation. Therefore, the mean conductivity that is calculated from the reconstructed distribution can be used to generate the new sensitivity matrix for the next data set as shown in (6), where $\left|m_{\mathrm{i}}\right|$ is the mean conductivity of reconstructed material, " $\alpha$ " is a convergence regulation parameter, 1 is the unit material vector, and $\left|m_{\mathrm{i}}^{\prime}\right|$ is the resulting value of mean conductivity that is used to generate the new sensitivity matrix.

$$
\left|m_{i}^{\prime}\right|=\alpha \cdot\left|m_{i}\right| \cdot 1 .
$$

The use of the mean conductivity in this way is novel and has been observed to be convergent in terms of (3)-(5), as suggested by Fig. 13, which demonstrates the reconstruction convergence where the initial reference material is far from the real material in terms of conductivity. The material distributions are the same as those used for the reconstructions shown in Fig. 10. The cost of this method is extra computation time caused by the need to recalculate the electric field and " $J$ " matrix at each step. This is acceptable in the present case, where the process dynamics are modest, and, consequently, the demands on data processing rates are relaxed. 

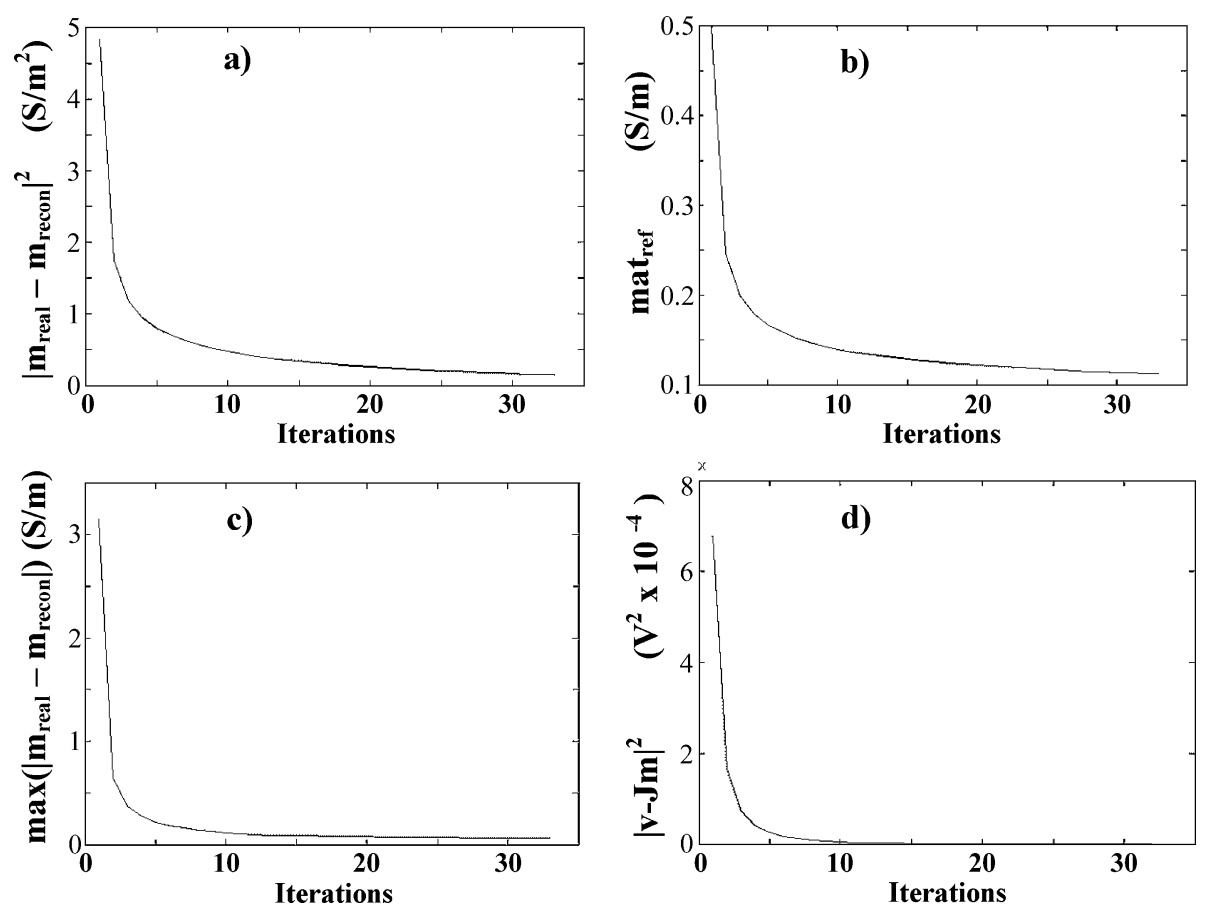

Fig. 13. Result of reconstruction with the evolving sensitivity matrix algorithm.

Level Measurement in $24 / 970$

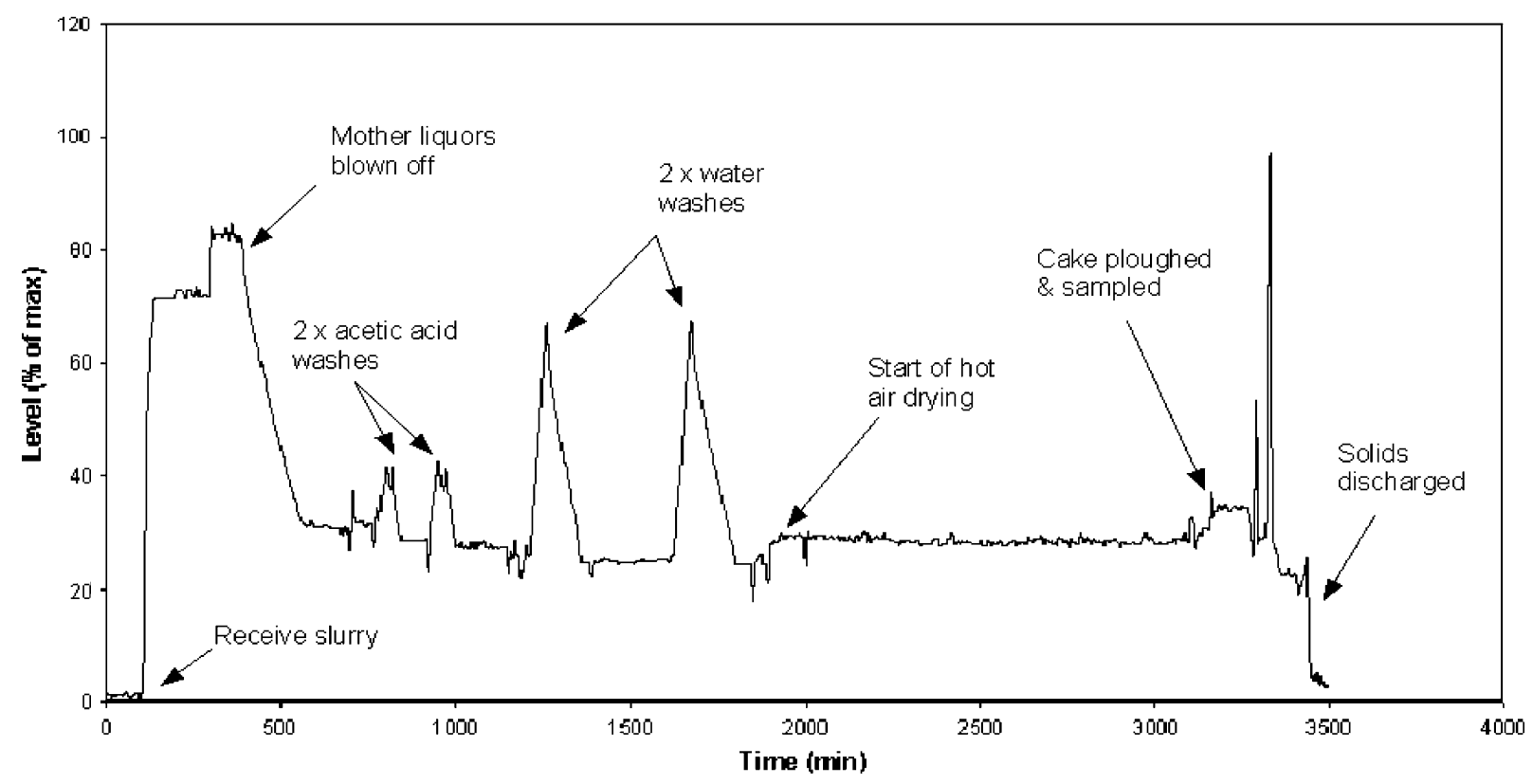

Fig. 14. Level indication in $36-\mathrm{m}^{3}$ filter across a complete batch cycle.

To speed up reconstruction and improve stability and convergence, estimation of the initial distribution is very important. This task can be achieved in many ways and the present proposal is to create a library of precalculated measurements corresponding to different material distributions. Every measurement result can be taken as a point in $N$-dimensional space, where $N$ is equal to number of single measurements. The library element that is closest to the measurement point that is obtained for an unknown material is used as the initial "guess."

\section{Process Understanding of the ImPedAnce Data}

As two-dimensional image reconstruction approaches were not directly applicable to electrodes mounted within the plane of the filter cloth, the initial interpretation of the data was carried out using statistical analysis of the impedance signals. The interpreted data have been related back to the on-line measurements obtained elsewhere in the vessel. The most useful of these is the slurry level measurement, which was delivered through an acoustic reflection instrument located in the roof of the vessel. 


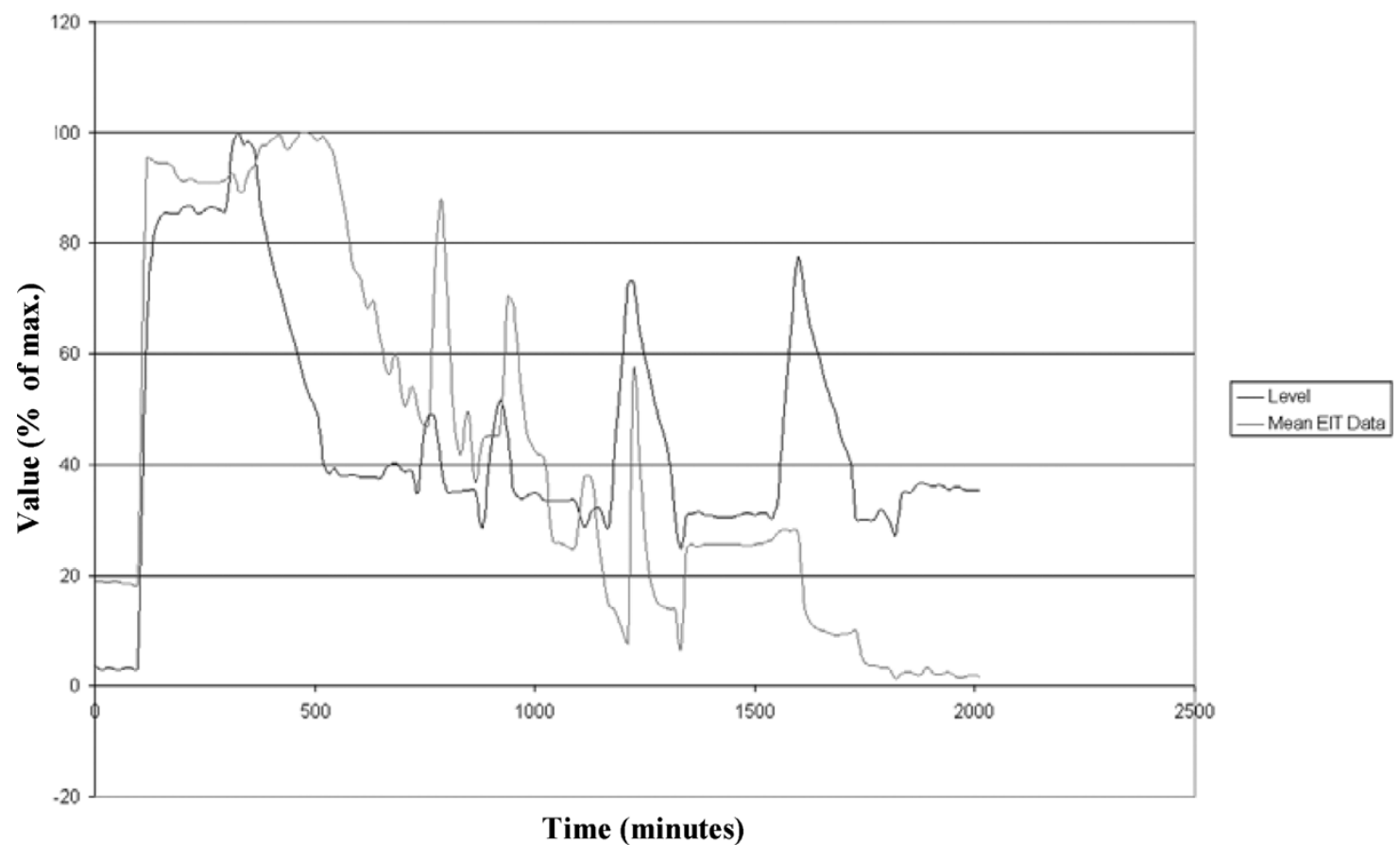

Fig. 15. Level indication overlaid with mean tomography signal.

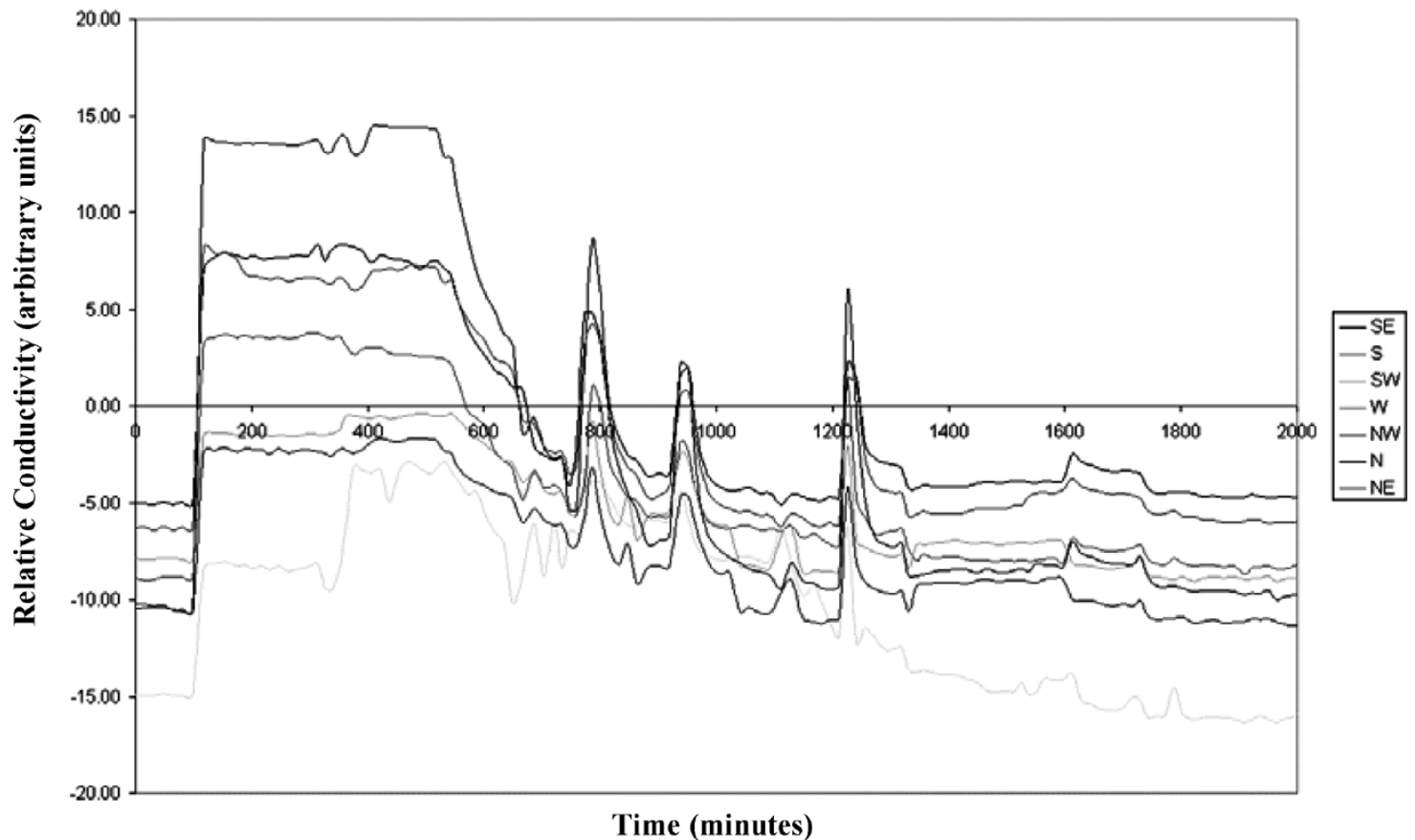

Fig. 16. Spatial distribution of tomography signals.

Since October 2000, the electrical impedance tomography instrument within the $36-\mathrm{m}^{3}$ filter has run for extended periods collecting data at $15-\mathrm{min}$ intervals using a $10-\mathrm{kHz}$ excitation signal. The following section provides an analysis of a single complete separation, washing and drying cycle. This information was collected in the first quarter of 2002 and is characteristic of that gathered from other batches. Fig. 14 graphs the level indication, taken at 5-min intervals, across a 3500-min batch sequence and is annotated with the principal process operations in progress at each stage.

In Fig. 15, the level data has been combined with the mean conductivity signal, derived directly from the EIT data on a 15-min cycle, and truncated to provide a more detailed view of the first $2000 \mathrm{~min}$ of processing. Clearly, there is good correlation between the tomography data and the level measurements. This run utilized the 226 independent measurements, of the possible 552 electrode drive and receive combinations, which were selected so as to provide a symmetrical distribution of field lines about the center point of the filter cloth.

By comparing these two trends, it can be readily seen that the mean tomography signal is distinct from the level data while also reflecting the general progress of the isolation and solvent displacement stages. The overall bias is toward a decrease in bulk conductivity, as the mother liquor is replaced by the wash 


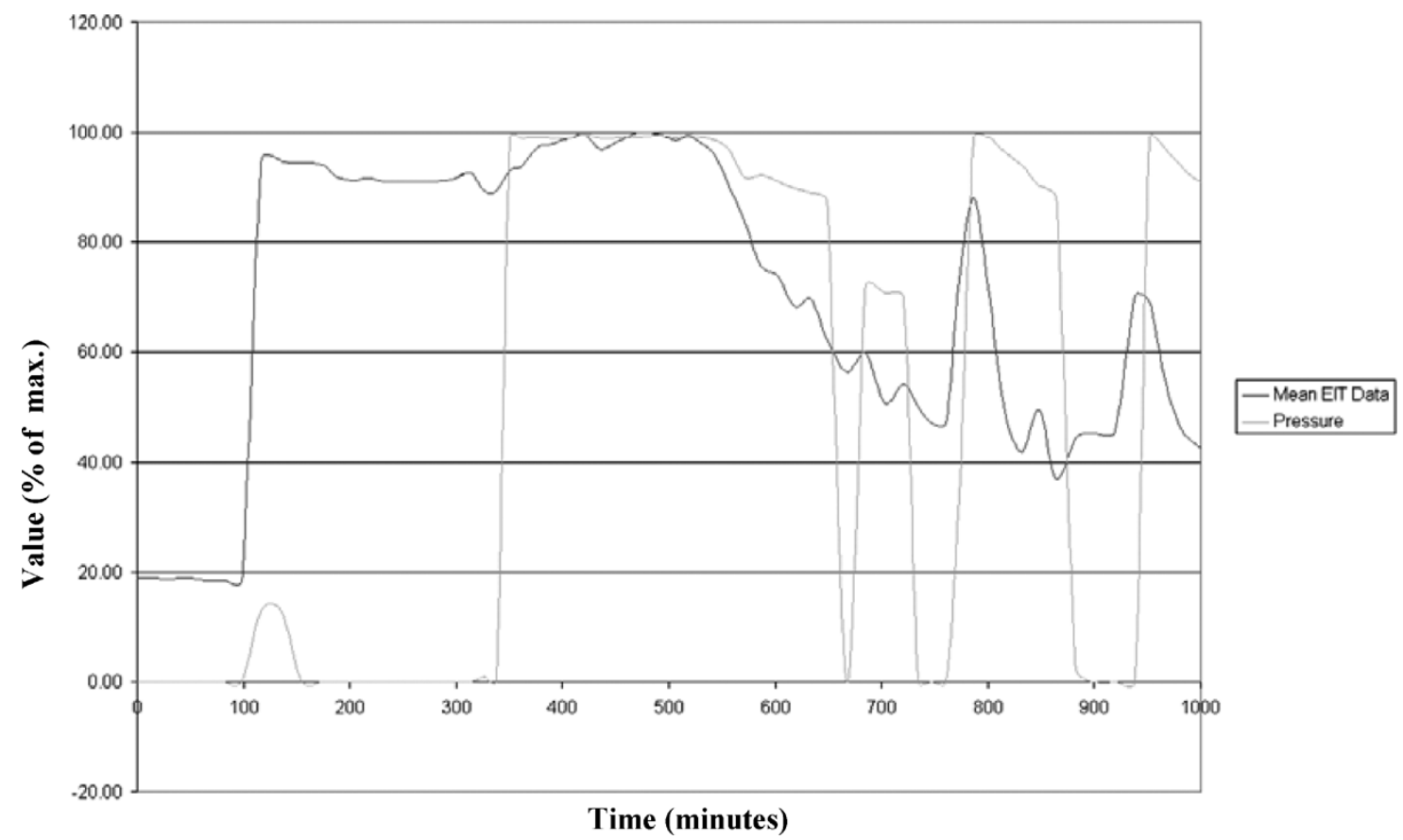

Fig. 17. Air pressure and mean tomography signal versus time.

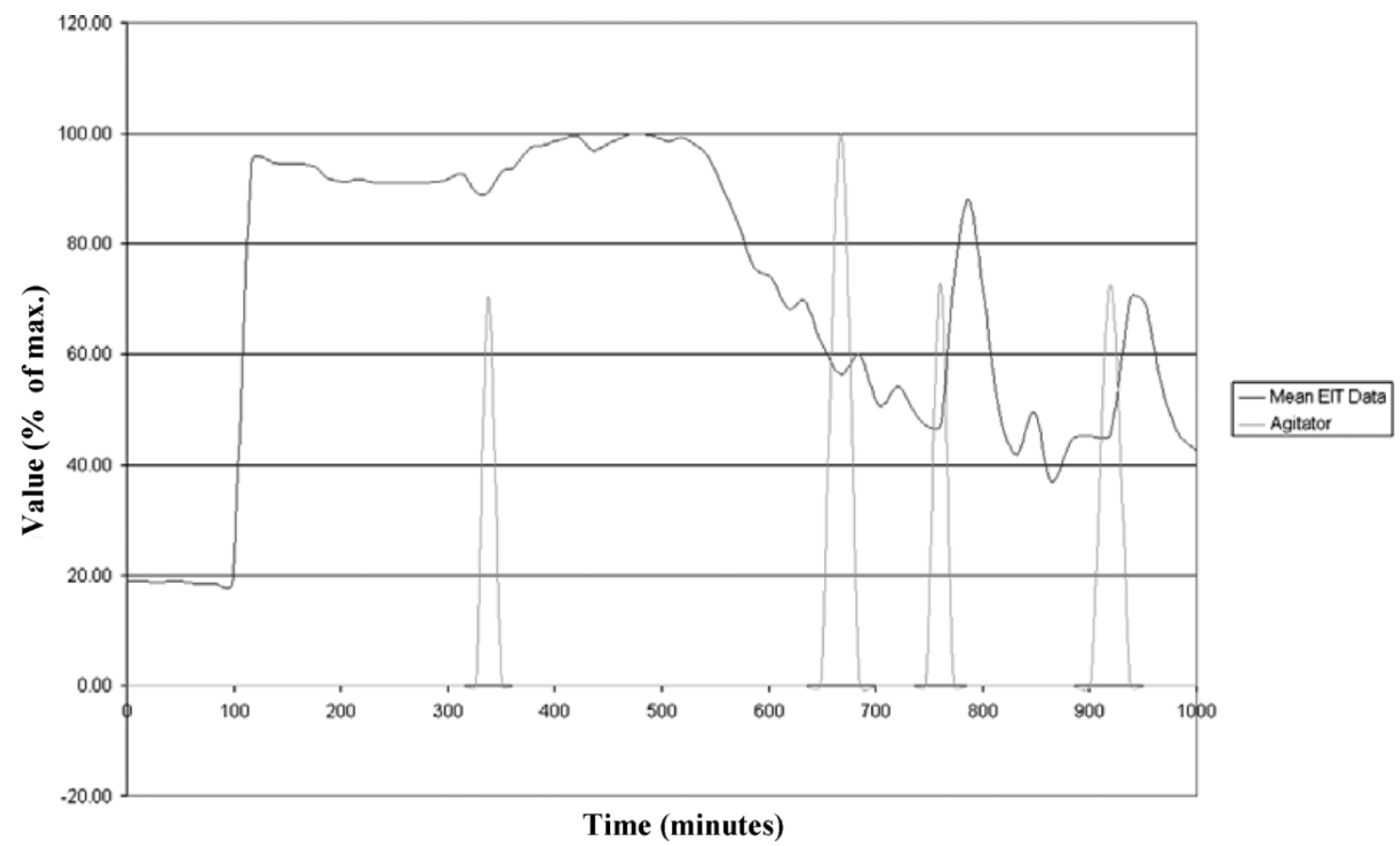

Fig. 18. Agitator load and mean tomography signal versus time.

solvents and as these are, in turn, forced out of the solid cake by the pressurized air. Without additional corroborative reference information, any further inference of the process state from these plots would be highly speculative.

Fig. 16 presents a more detailed view of the variation in the tomography signal obtained across the solid volume by selecting eight pertinent groupings of drive and receive electrodes taken symmetrically across the filter cloth. These regions correspond to the cross-section of the filter being divided into sectors of $\mathrm{N}$, NE, E, SE, S, SW, W, NW, and N. The graph's time window is identical to that in Fig. 15, and the data scales have been arbi- trarily chosen to allow the readings to be fitted on to one plot. As it is the relative deviation in the signals which is of interest in this case, the absolute scaling is not critical. From these regional plots, it can be seen that the tomography data does indicate that the spatial conductivity distribution, inferred from the tomography data, is not even throughout the filtered solid, the implication being that the batch's solvent composition or solids packing is inhomogeneous. As with the averaged tomography data, additional laboratory-based experimentation under controlled conditions would be required to quantify this measure of cake composition. It should be noted that the batch under 


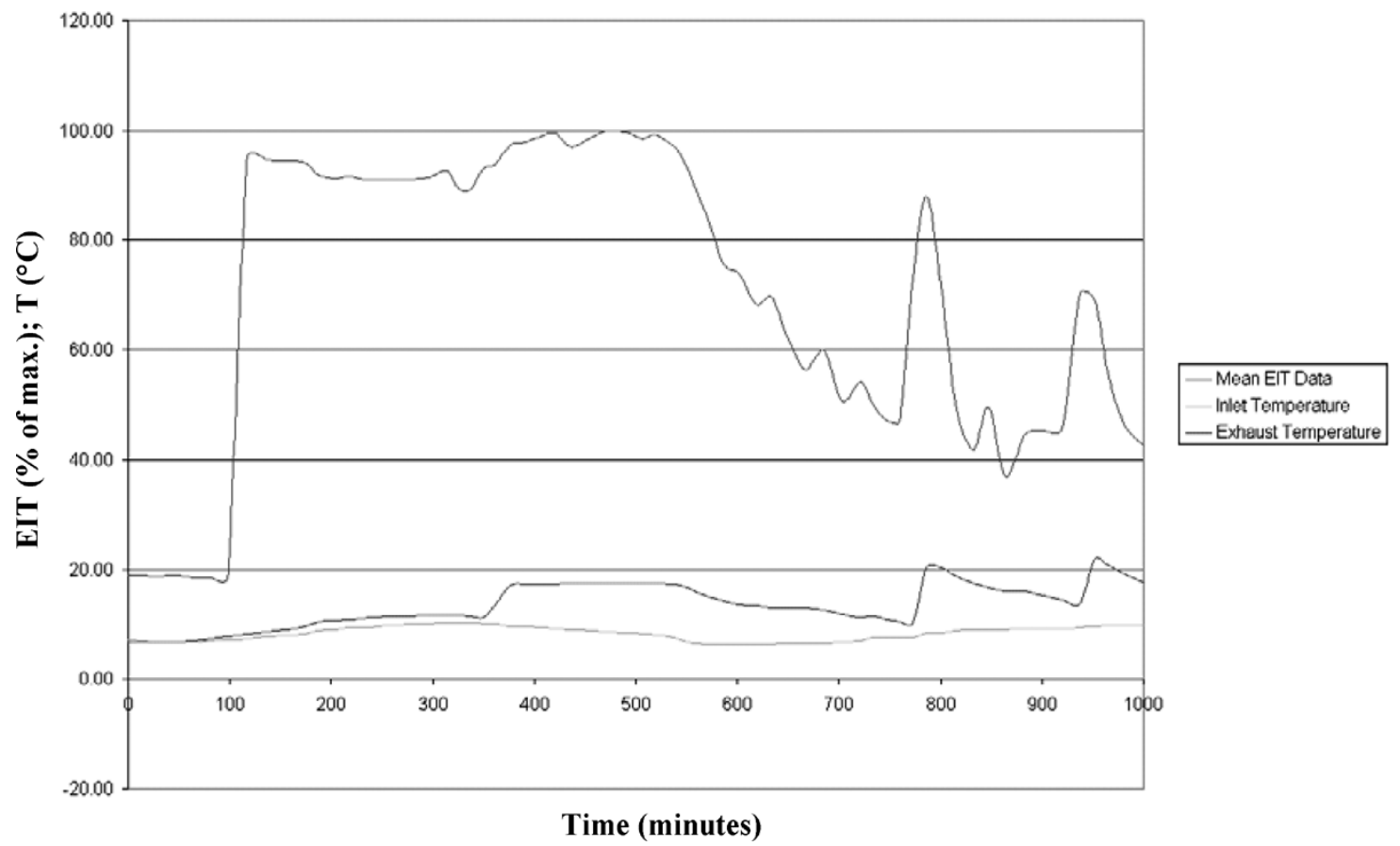

Fig. 19. Air temperature and mean tomography signal versus time.

examination passed the manual single-point moisture analysis carried out at the end of drying.

Figs. 17-19 are included to illustrate the effect of other external factors on the tomography measurements, namely agitator rotation, air pressure, and temperature alterations. To simplify these diagrams, only the first 100 -min period relating to the initial charging of the slurry, the crude removal of the mother liquor, and the acetic washes have been extracted from the total data set. With reference to Fig. 17, when the air pressure is first applied to the raw slurry, after $340 \mathrm{~min}$, there is no appreciable immediate effect on the tomography signal. This indicates that the system has little or no direct cross sensitivity to the headspace pressure. The correlation between these variables which does occur from 500 min onward is most probably a secondary effect arising from the mother liquor and acetic acid wash solvent flows. The spike in the agitator's drive current, shown in Fig. 18 after 310 min, corresponds to the crude slurry being uniformly blended by the anticlockwise rotation of the paddles. It is interesting to note that, though this is a large earthed component, the empirical data would point toward it having little effect on the measured mean conductivity, possibly due to its location at this time being mid height in the vessel or its rapid rotation, with respect to the frame capture rate of the instrument. The second agitator load, which occurs after $650 \mathrm{~min}$, relates to the smoothing of the surface of the filter cake by the slow rotation of the blades in contact with its upper strata. Again, only minor variations in the tomography signal arise, which are probably due to the redistribution of the solids rather than a direct effect from the circulating paddles. The last two excursions in the agitator current are linked to the feeding of the acetic wash solvents and the following increases in the tomography signal are almost certainly due to the acid passing through the cake. The last plots, given by Fig. 19, depict the mean tomographic signals alongside the incoming and vented air tempera-

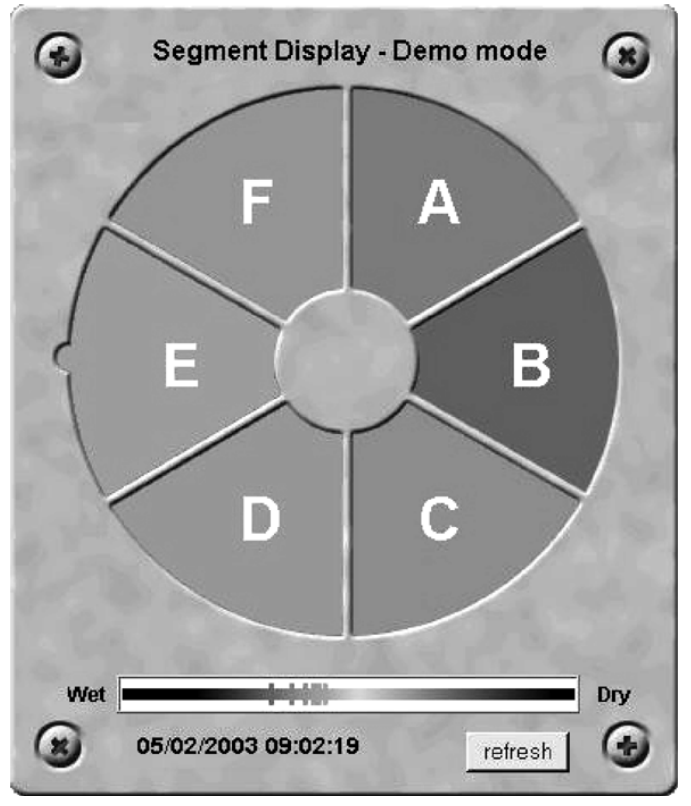

Fig. 20. Cross-sectional intranet image of the $36-\mathrm{m}^{3}$ filter showing the six sectors.

tures. The total dynamic range of the latter being approximately $10{ }^{\circ} \mathrm{C}$ for this time slice. As the inlet air temperature is fairly consistent while the exhaust shows a degree of increase during mother liquor removal and the acid washes, there is a suggestion that an exothermic process is taking place or the bulk material is gaining kinetic energy from the agitator. Despite this, the tomography signals appear to be suitably independent of these mechanisms to offer distinct process information.

Data captured using the tomography system are preprocessed before being displayed in real time on a website which is accessible through the Syngenta intranet LAN. The displayed data 


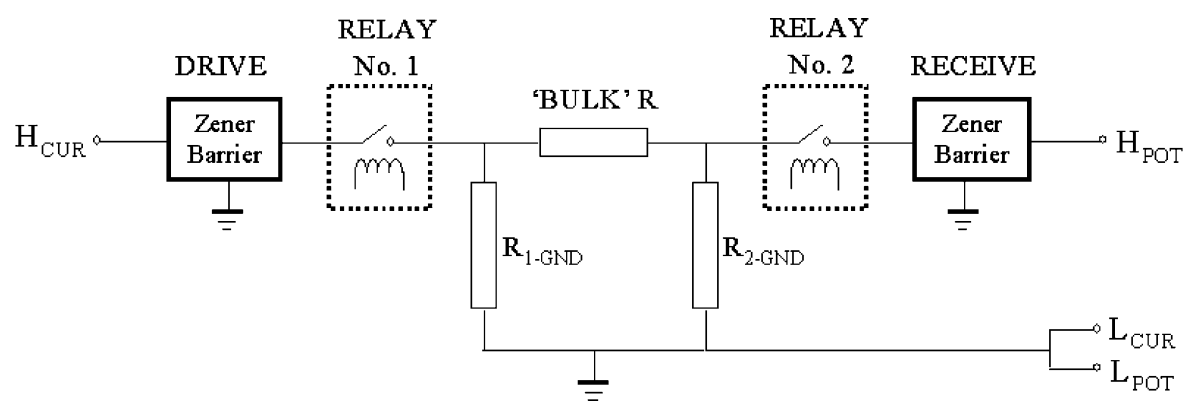

Fig. 21. Schematic of the three-terminal configuration.

gives plant personnel the ability to monitor individual process steps. This will ultimately enable an optimization of individual steps with the main specific benefits of improving both washing efficiency and the measurement of product homogeneity.

The preprocessing is performed by bespoke software, which calculates the mean measurements within the $36-\mathrm{m}^{3}$ pressure filter for 1) all signals for each working electrode and 2) groups of electrodes. The grouped electrodes give six in-plane sectors and are grouped in such a way as to give rise to approximately equal sector areas with each sector considering measurements from at least five working electrodes. The mean measurements consider a scaling to calibration data for maximum wetness and dryness within the filter for known conditions. In effect, this allows for a simple preliminary measurement of moisture content within the cake relative to the calibration data. The intranet website displays the data continuously in two forms, first as a segment display and, second, as histograms for each sector giving an approximate relative dryness level over a time period of almost four days maximum. These histograms are shown simultaneously alongside conventional plant data, which give the current and previous process steps within the displayed time period. All the displayed information can be saved on request along with text notes added by plant personnel as to indicate any abnormalities with the current batch. Routine inspection of the website, has suggested that the estimated "dryness" is not even throughout the filter cake for various process steps over a number of separate batches. This can be seen in segment display example shown in the Fig. 20. However, it should be noted that for a complete understanding of cake dryness, further laboratory-based investigations are required to relate the measured impedance values to typical solid conductivities during the filtration process.

\section{DISCUSSION}

The work program has exposed some unexpected issues relating to the use of the impedance analyzer in the way described. These are due to the employment of the intrinsically safe circuitry and, in particular, the presence of ZB modules to limit voltage excursions. These introduce considerable parasitic capacitance to ground that acts as a leakage path for the injected current which, of course, becomes more significant with increases in frequency. Consequently, this has introduced difficulties in reconstructing electrical impedance images of the filter from the measured data. A schematic model of the three-terminal connection configuration between the LCR meter and the filtration unit, for the case of electrode 1 driving and electrode 2 receiving, is shown in Fig. 21. The model assumes that the bulk material in the filter acts as a pure resistance "BULK R" between the driving and receiving electrodes plus resistances to ground, " $\mathrm{R}_{1-\text { GND" }}$ " and " $\mathrm{R}_{2-\text { GND }}$ " which are strongly affected by the close proximity of the electrodes to the filter-cloth hold-down bars. In addition, two further current paths to ground exist via the parasitic capacitance in the $\mathrm{ZB}$ modules.

Laboratory studies have investigated the effect of the shunting capacitance by taking measurements with RMS voltmeters alongside the LCR meter and using resistors arranged to implement the model shown in Fig. 21. It should be acknowledged that, although the configuration of the LCR meter is outside the manufacturer's specification, it is effectively only being used to provide measurements of injected current and measured voltage and not true impedance. In the experiments, the bulk resistance was varied from $10 \Omega$ to $10 \mathrm{M} \Omega$

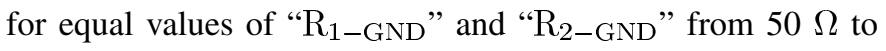
$500 \mathrm{k} \Omega$. The measurements confirmed that the three-terminal configuration provides a good measurement of the voltage at the receiving electrode via the " $\mathrm{H}_{\mathrm{POT}}$ " terminal of the LCR meter over the complete range and the effects of the "receive" $\mathrm{ZB}$ and " $\mathrm{R}_{2-\mathrm{GND}}$ " can be ignored. However, the experiments confirmed that direct measurement of the injection current from the driving electrode is more problematic due to the "drive" ZB (Pepperl and Fuchs ZB966). The capacitance to ground of this barrier was measured to be approximately $2 \mathrm{nF}$ and arises as a consequence of the large $\mathrm{p}$ - $\mathrm{n}$ junction areas of 12 internal zener diodes.

Attention was, therefore, focussed on the effect of the barriers during two extreme phases of the filtration process, namely washing and drying when the resistances to ground are low and high respectively. For the washing phase, the injected current is about $450 \mu \mathrm{A}$ and is, essentially, independent of frequency. The percentage current loss due to the ZBs is relatively small for the frequency range employed in the project and is shown in Fig. 22. However, for the drying phase, the injected current varies with frequency from about $50 \mu \mathrm{A}$ at $10 \mathrm{kHz}$ to $450 \mu \mathrm{A}$ at $100 \mathrm{kHz}$. Over this range of frequencies, the current loss varies from about $50 \%$ to $90 \%$, respectively, of that suggested by the LCR meter, and, clearly, this presents difficulties for image reconstruction, as the measured value of current that is injected to the vessel is not accurately known. The currents that were observed during these laboratory tests are similar to those that were observed during actual measurements on the pressure filter. 


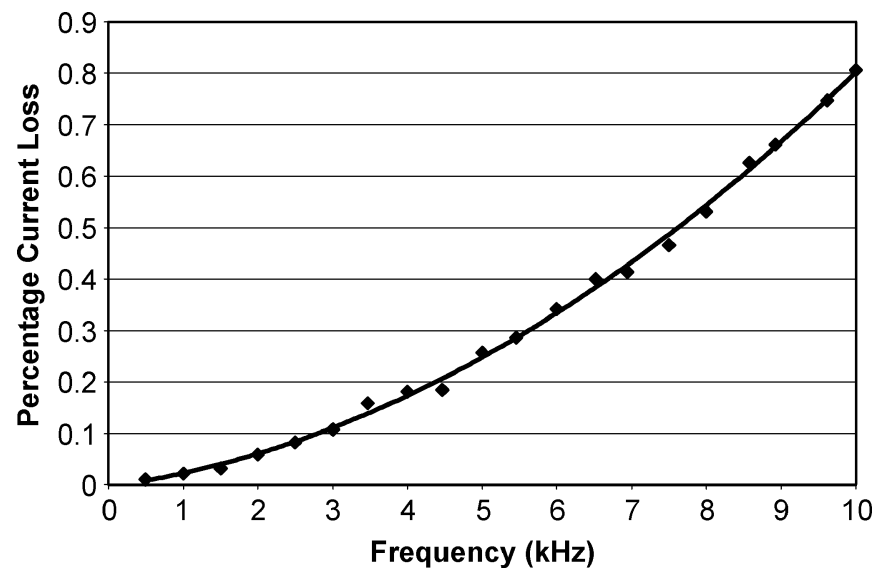

Fig. 22. Percentage loss of injected current due to the drive ZB.

Although not a significant issue for washing phases, the current loss must be addressed for successful image reconstruction during the drying stages of the filtration process. Consequently, future work is considering compensating for the current loss by directly modeling the barriers into the FEM in order to achieve a more accurate forward solution.

\section{SUMMARY}

Intrinsically safe EIT utilizing electrode architectures and materials which are compliant with the process, as opposed to convenient for image reconstruction, has been demonstrated for the first time within metal-walled industrial production units. Two instruments are collecting data, on a regular basis, from production pressure filters at Syngenta in Huddersfield. With the introduction of "live" results on the company intranet plant, operators are now able to integrate the resulting data into the decision-making process regarding the status of each batch. This offers immediate benefits in terms of efficiency, waste, quality, and safety.

Initially, pressure filtration processes have been monitored using a simple approach which averages tomography signals from groups of electrodes that are located in a particular segment of the filter. The features detected, using basic statistical tools, indicate that EIT can provide new insight into pressure filtration and, by inference, other heterogeneous batch processes. This information has been correlated with the conventional plant measurements and successfully relayed in real-time across an intranet system, to allow further investigation by the manufacturing plant operations and process development personnel.

A method of reconstructing 3-D images for any electrode arrangement and vessel structure has been developed by translating the EIDORS 3-D software toolkit and the conducting boundary strategy to the subject processes. As a consequence, a generic method has now been realized to computer model the effectiveness of any process compliant electrode array, irrespective of its symmetry. The implications of this are far reaching and lead to the possibility of utilizing the existing furniture within a process unit, such as dip legs, agitator blades, and baffle plates, to act as EIT electrode arrays in a 3-D imaging system. By electrically isolating these components from earth through retrofitting insulating spacers, while also maintaining their static potential at a safe level by means of the I.S. interface, the economic and practical barriers to broad uptake of EIT by industry can be removed.

In addition, the EIDORS 3-D reconstruction approach has been further enhanced by the introduction of dynamic sensitivity maps that evolve according to the conductivity changes. In this way, the degradation of the images resulting from dramatic changes in the pervading conductivity within the process may be alleviated. Currently, this quantitative reconstruction technique has only been applied to simulated data. A number of issues arise for real measurements from the filter. First, the value of the contact impedance depends on both the electric current and the chemical properties of the material. Another important problem arises due to the intrinsically safe hardware. When the filter cake is dry, the measurements show negative readings. Laboratory investigations have revealed that this is due to the shunt capacitance presented by the ZB modules which leads to errors in the measurements recorded by the LCR meter. This should be compensated for in the reconstruction algorithm. Finally, the lifetime of the relays has caused some problems. The suggested operating lifetime is five million cycles. For our system, with 24 electrodes taking measurements every $15 \mathrm{~min}$, each relay will switch approximately one million times per year. Consequently, in a relatively short time, the relays are approaching the end of their useful life. The possibility of replacing these with certified, solid-state switches is being investigated. On-going work is considering new I.S. instruments that can overcome these limitations.

\section{REFERENCES}

[1] D. S. Vlaev, M. Wang, T. Dyakowski, R. Mann, and B. D. Grieve, "Detecting filter-cake pathologies in solid-liquid filtration: semi-tech scale demonstrations using electrical resistance tomography," Chem. Eng. J., vol. 77, no. 1-2, pp. 87-92, 2000.

[2] E. L. Yuen, D. Vlaev, R. Mann, T. Dyakowski, B. D. Grieve, and T. A York, "Applying electrical resistance tomography to solid-fluid filtration processes," in Proc. World Filtration Congr. 8, Brighton, U.K., 2000, pp. 3-7.

[3] T. A. York, Q. Smit, J. L. Davidson, and B. D. Grieve, "An intrinsically safe electrical tomography system," in Proc. IEEE Int. Symp. Industrial Electronics, vol. 2, Jun. 2003, pp. 946-951.

[4] B. D. Grieve, Q. Smit, R. Mann, and T. A. York, "The application of electrical resistance tomography to a large volume production pressure filter," in Proc. 2nd World Congr. Industrial Process Tomography, Hanover, Germany, Aug. 2001, pp. 175-182.

[5] B. D. Grieve, J. L. Davidson, R. Mann, W. R. B. Lionheart, and T. A York, "Process compliant electrical impedance tomography for widescale exploitation on industrial vessels," in Proc. 3rd World Congr. Industrial Tomography, Banff, Canada, 2003, pp. 806-812.

[6] W. R. B. Lionheart, "Reconstruction algorithms for permittivity and conductivity imaging," in Proc. 2nd World Congr. Industrial Process Tomography, Hannover, Germany, 2001, pp. 4-11.

[7] N. Polydorides and W. R. B. Lionheart, "A MatLab based toolkit for three-dimensional electrical impedance tomography: a contribution to the EIDORS project," Meas. Sci. Technol., vol. 13, no. 12, pp. 1871-1883, 2002.

[8] T. A. York, "Status of electrical tomography in industrial applications," J. Electron. Imag., vol. 10, no. 3, pp. 608-620, 2001.

[9] M. Wang, F. J. Dickin, and R. A. Williams, "Modeling and analysis of electrically conducting vessels and pipelines in electrical resistance process tomography," Proc. Inst. Elect. Eng., vol. 142, no. 4, pp. 313-322, 1995.

[10] R. Garside, Intrinsic Safety, Electrical Apparatus and Hazardous Areas, 3rd ed. Aylsebury, U.K.: Hexagon Technology, pp. 230-256.

[11] P. P. Sylvester and R. F. Ferrari, Finite Elements for Electrical Engineers, 3rd ed. Cambridge, U.K.: Cambridge Univ. Press, 1996.

[12] NETGEN: 3-D Mesh Generation Software, J. Schöberl. http://www.hpfem.jku.at/netgen [Online] 
Trevor A. York (M'97) received the B.Sc. and Ph.D. degrees in physics from the University of Manchester Institute of Technology (UMIST), Manchester, U.K., in 1979 and 1982, respectively.

Since 1985, he has been with the Department of Electrical Engineering and Electronics, UMIST, specializing in microelectronics. He was promoted to Professor in 2004. Currently, he is exploring the design of intrinsically safe electrical tomography systems for monitoring industrial filtration processes and trials are underway on production vessels. Work to realize miniaturized electrical tomography systems is now proceeding with an application in the automobile industry. He has published 100 academic papers, and his main research activity is in industrial process tomography systems with particular emphasis on microelectronic applications.

Dr. York is a Fellow of the Instiutute of Electrical Engineers and the Institute of Learning and Teaching in the U.K.

John L. Davidson received the B.Sc. degree in applied physics and the Ph.D. degree from Sheffield Hallam University, Sheffield, U.K., in 1993 and 1997, respectively.

For four years, he was a Postdoctoral Researcher with the Department of Electronics and Computer Science, University of Southampton, Southampton, U.K., developing a novel sensing arrangement for the mapping of surface charges on insulating surfaces. The technique was successfully applied for the first accurate measurements of electrostatic charge transfer to living insects generated by walking on surfaces and was featured in New Scientist magazine. Since 1992, he has been with the School of Electrical and Electronic Engineering, University of Manchester, Manchester, U.K. He has authored and coauthored almost 20 academic publications. His research interests are mainly associated with industrial process tomography, with particular emphasis on both application development and image reconstruction.

Dr. Davidson is a member of the Institute of Physics.
Lukasz Mazurkiewich received the M.Sc. degree in computer science from the Technical University of Lodz (TUL), Lodz, Poland, in 2001, where he is currently pursuing the Ph.D. degree in 3-D imaging and flow monitoring for process tomography.

Since 2001, he has been with the Computer Engineering Department, TUL. He has published 20 academic papers.

Reginald Mann is a Professor of chemical reaction engineering at the University of Manchester, Manchester, U.K., where he has been a member of the teaching staff since 1972. His principal research interests are in applied catalysis and mixing in chemical reactors. He has pioneered the use of process tomography so as to quantify the segregated concentration fields that arise in chemical reactors which are not well mixed. Such imperfect mixing can profoundly distort the chemical yield achieved. He is also seeking to apply tomography for validation of computational fluid dynamics (CFD) for chemical reactors. He has published more than 100 technical papers and has supervised over 40 doctoral candidates.

Bruce D. Grieve received the B.Sc. degree in electronic engineering from the University of York, York, U.K., and the Ph.D. degree from the Department of Chemical Engineering, University of Manchester, Manchester, U.K., in 1988 and 2002, respectively.

He is a Chartered Research Engineer with 15 years of experience in the chemical, pharmaceutical, and biotechnology sectors. He is currently a Leading Scientist within Syngenta's Global Specialist Technology Group, Huddersfield, U.K. His research interests focus on identifying and migrating emerging sensors, instrumentation, and data-mining technologies toward novel applications. A core research interest of his is the development of multifrequency, noninvasive, electrical impedance imaging through amalgamating electronics, chemical engineering, and electrochemistry disciplines with 3-D finite-element reconstruction techniques. Other research interests include vibrational spectroscopy, acoustic monitoring of crystallization and nucleation, multivariate chemometric data analysis, and MEMS design for self-assembly, nano-colloidal processing and purification. 